\title{
Reliability of 3D Gait Data across Multiple Laboratories
}

\section{$\underline{\text { Authors }}$}

Kenton Kaufman, Ph.D., P.E., Biomechanics-Motion Analysis Laboratory, Mayo Clinic, Rochester, MN

Emily Miller MS; Motion Analysis Laboratory, Mayo Clinic, Rochester, MN

Trevor Kingsbury MS; Naval Medical Center San Diego, CA

Elizabeth Russell Esposito PhD; Center for the Intrepid, JBSA Fort Sam Houston, TX

Erik Wolf, PhD; Walter Reed National Military Medical Center, Bethesda, MD

Jason Wilken, PhD, PT; Center for the Intrepid, JBSA Fort Sam Houston, TX

Marilynn Wyatt, MA, PT; Naval Medical Center San Diego, CA

$\begin{array}{ll}\text { Corresponding author: } & \text { Kenton R. Kaufman, Ph.D., P.E. } \\ & \text { Biomechanics-Motion Analysis Laboratory } \\ & \text { Charlton North L-110L } \\ & \text { 200 First Street SW } \\ & \text { Rochester, MN 55905 } \\ & \text { Email: kaufman.kenton@ mayo.edu }\end{array}$

Keywords: Gait, Motion Analysis, Reliability, Reproducibility of Results

Source of support: DOD 731743-1, DOD Defense Health Programs/Center for Rehabilitative Sciences Research, Grant \# HU0001-11-1-0004

\begin{abstract}
The aim of this study was to analyze the repeatability of gait analysis studies performed across multiple trials, sessions, and laboratories. Ten healthy participants (6 male/4 female, mean age of 30 , mean BMI of $24 \mathrm{~kg} / \mathrm{m}^{2}$ ) were assessed in 3 sessions conducted at each of the three Centers of Excellence for Amputee Care within the Department of Defense. For each test session, kinematic and kinetic parameters were collected during five walking trials for each limb. One independent examiner at each
\end{abstract}


site placed markers on the subjects. Biomechanical data were collected at two walking speeds: selfselected and Froude speed. Variability of the gait data was attributed to inter-trial, inter-session, and inter-lab errors for each subject. These error sources were averaged across all ten subjects to obtain a pooled error estimate. The kinematic errors were fairly consistent at the two walking speeds tested. Median inter-lab kinematic errors were <5.0 degrees (median 2.3 degrees) for all joint angle measurements. However, the kinetic error differed significantly between walking speeds. The median inter-lab kinetic error for the self-selected speed was $0.112 \mathrm{Nm} / \mathrm{kg}$ (ICR 0.091-0.184) with a maximum of $0.226 \mathrm{Nm} / \mathrm{kg}$. The errors were greatly reduced when the subjects walked at their Froude speed. The median inter-lab error was $0.048 \mathrm{Nm} / \mathrm{kg}$ (ICR $0.025-0.078$, maximum 0.086). These data demonstrate that it is possible to get reliable data across multiple gait laboratories, particularly when gait speed is standardized across testing sessions. A key similarity between sites was the use of identical anatomical segment definitions for the respective gait models. 


\section{INTRODUCTION}

Gait studies performed on the same individual in presumably identical circumstances do not necessarily yield identical results. These differences may be attributed to errors inherent in every test procedure. Factors that affect the outcome of a gait study cannot all be completely controlled. Variability in 3D gait data can come from both intrinsic and extrinsic factors. Extrinsic factors that add to this variability come from differences between data collection sessions and differences between researchers. For example, extrinsic factors may result from differences in positioning the reflective markers [1]. Intrinsic factors that affect error may include intra-individual variations that arise naturally from trial-to-trial and subject-to-subject, neither of which can be reduced [2]. For example, age and walking speed can both contribute to the natural variability of gait between subjects [3-5]. In order to assess these factors, several walking trials are typically collected during the same session to provide a measure of the data variability. In healthy subjects it is generally thought that five trials are sufficient to obtain reliable data [6] [7].

Many different factors may contribute to the total variability in gait data including the operator, equipment used, and equipment calibration. Therefore, it is not surprising that recent studies have found that the largest sources of extrinsic variability come from tests across different laboratories. Noonan et al.[8] tested 11 patients with cerebral palsy at four separate gait analysis laboratories and found that at least 5\% of gait cycle could vary among laboratories by at least 21 degrees. The authors also found a 27 degree average difference in static range of motion across laboratories. Most importantly, the results of the gait analysis generated considerably different treatment recommendations across four sites. Gorton et al. [9] evaluated one healthy subject at twelve Shriner's Hospitals and found an average maximum difference of 15 degrees across sites. Variability was also high between subsequent sessions at a single site with 5 of 8 gait parameters significantly different. Most recently, Benedetti et al. [10] assessed the consistency of one healthy subject's gait at seven different laboratories and concluded that joint angles 
and moments were generally consistent, with the exception of hip and knee joint rotations, but there were systematic errors in the data across sites, particularly in hip and knee powers. These studies raise questions about comparing gait data between different laboratories and, although some of the results are promising, concerns about inter-lab reliability still remain.

Data reliability is of utmost importance when attempting to share data across sites to evaluate patient progress or to pool research data for comparative effectiveness studies. Therefore, the goal of this study was to collect consistent 3D gait data across three motion analysis laboratories. Prior work has identified five degrees as an upper end criterion of minimal detectable change for almost all kinematic measures [20]. There isn't an establised criterion for kinetic data.We hypothesized that with appropriate calibration of the equipment and uniformity of the underlying biomechanical model it would be possible to obtain reliable measurements. The results of this study are important because measurement consistency across sites participating in multi-center research studies directly influences required sample size, level of detectable difference, and statistical power.

\section{METHODS}

Ten subjects (six male/four female, age $30 \pm 6$ years, BMI $24 \pm 4 \mathrm{~kg} / \mathrm{m}^{2}$ ) without any neuromuscular impairment were studied. All subjects were screened by a licensed physical therapist. Inclusion criteria were individuals between the ages of 10-45 years who had normal muscle strength and range of motion of the lower extremities with no neuromusculoskeletal deficits. Individuals were excluded from the study if they had a major orthopedic injury such as a meniscal tear, ligament tear, or recent sprain or fracture within the last year or any surgery to the lower extremities. Individuals who were pregnant were also excluded. The experimental protocol was approved by the Naval Medical Center San Diego Institutional Review Board as well as the Human Research Protection Office, U.S. 
Army Medical Research and Materiel Command. Subjects provided written informed consent before participating in this study.

Data were gathered at the three Centers of Excellence for Amputee Care within the Department of Defense: Comprehensive Combat and Complex Casualty Care (C5) at the Naval Medical Center San Diego (NMCSD), California (MTF 1), Military Performance Laboratory, Center for the Intrepid (CFI) at San Antonio Military Medical Center, JBSA Fort Sam Houston, Texas (MTF 2), and the Center for Performance and Clinical Research Amputee Service, Walter Reed National Military Medical Center (WRNMMC), Bethesda, Maryland. All centers have gait analysis laboratories that are used for routine clinical care and research. The motion capture systems and the number of cameras used differed at the three sites (Table 1). All sites were using force plates from the same manufacturer (AMTI, Watertown, MA) but the model, number of force plates, and data collection rate differed at all three sites (Table 1).

Prior to gait data being collected at any of the sites, a set of mechanical tests were performed to assure that all equipment was operating in a consistent manner. The quality of the data for the motion capture system was assessed using a rotating frame with six markers attached to the frame and one stationary marker on the base of the rotating frame [11]. Distance between markers was compared to measured distances and errors were evaluated. The errors differed by $<2.3 \mathrm{~mm}$ across all laboratories for marker separations which ranged from $34 \mathrm{~mm}$ to $64 \mathrm{~mm}$. Next, the integration of the force plate with the motion capture system was tested by applying a force to the force plate using a rod with markers attached[12]. The alignment of the force vector and the calibration stick were assessed for colinearity. The center of pressure from the two methods differed by a maximum of $5.4 \mathrm{~mm}$. Finally, a mechanical leg and pelvis were used to attach markers and assess the output of the underlying biomechanical model. The simulated leg was rotated \pm 10 and 20 degrees at the hip, knee, or both. The joint angles calculated by the software were compared to the experimental data and errors were 
calculated. The RMS error was 3 degrees at the hip and 5 degrees at the knee for single joint rotations. The RMS error increased to 5 degrees at the hip and 7 degrees at the knee for combined hip and knee rotations. Through this testing, a force plate error was detected at one MTF and a calibration error was identified at a second MTF. Both errors were resolved prior to biomechanical data collection.

The subjects were studied at all three sites within a twelve month period. The protocol for data collection was similar at all sites. Markers were placed directly on the skin and anthropometric data were collected by one examiner at each site. Each lab used its own tracking marker set (Figure 1) and standard operating procedures for data collection. Three-dimensional marker trajectory data were collected at $120 \mathrm{~Hz}$ and filtered using a fourth-order Butterworth low-pass filter with a cutoff frequency of $6 \mathrm{~Hz}$. The tracking markers followed a modified Helen Hayes model [13] or a Cleveland Clinic model [14]. Importantly, the underlying biomechanical model did not differ across sites. The hip joint center was approximated using the Bell model $[15,16]$. Medial and lateral femoral condyle calibration points and medial and lateral ankle calibration points were used for establishing the knee and ankle joint centers. Depending on the lab, some of these markers were then removed for the walking trails. Data were collected while the subject was wearing athletic shorts and the same athletic-type shoes for all trials. All of the markers associated with the foot were placed on the outside of the subject's shoes. Following the application of the reflective marker set, data were collected while the subject walked over level ground at a self-selected and standardized Froude (FR) speed. The Froude speed was standardized by leg length and a Froude number of 0.16 , where $\mathrm{FR}=\mathrm{s}^{2} / \mathrm{gL}$, where $\mathrm{s}$ is the walking speed, $\mathrm{g}$ is the gravitational constant, and L is the leg length [17]. Ground reaction force data were collected from force plates at a sampling rate of $600 \mathrm{~Hz}$ or higher (Table 1). Data from these force plates was timesynchronized with the motion cameras. Data were processed with Visual3D (C-Motion, Germantown, Maryland). At each site and at both speeds lower extremity joint angles and moments, and pelvis and 
foot segment angles were calculated across five strides to assess inter-trial reliability. Three separate data collection sessions were performed at each site to assess inter-session reliability. The order in which each subject visited the three labs was not randomized and was based on his or her availability to travel. Data at each site were de-identified and sent to an individual evaluator for analysis.

Variability of the gait data was attributed to intra-trial, inter-session, and inter-lab errors. Intertrial, inter-session, and inter-laboratory error was calculated for each subject using established techniques [2]. These error sources were then averaged across all ten subjects to obtain a pooled error estimate. The data were checked for normality using an Anderson-Darling normality test. All summary data were not normally distributed, so the median and inter-quartile range (ICR) was used to describe the data.

\section{RESULTS}

The inter-trial, inter-session, and inter-laboratory errors in lower extremity kinematics and kinetics were calculated for every point in the gait cycle for each subject (Figure 2). Reliability of each variable was expressed by the standard deviation of its measurement. The inter-trial error represents the variability of the subject's gait and serves as an appropriate reference for comparisons. The inter-session error was larger than the inter-trial error. The inter-lab error was larger than the inter-session error. The kinematic errors were fairly consistent across the entire gait cycle and were generally less than 5 degrees. The kinetic errors were slightly larger at toe off than during other portions of the gait cycle.

The walking speed differed for the two testing conditions. The self-selected walking speed was $1.43 \pm 0.14 \mathrm{~m} / \mathrm{sec}(95 \% \mathrm{CI}$ of mean $=1.38-1.48$, range $1.20-1.74)$. The standardized (Froude) walking speed was $1.20 \pm 0.04 \mathrm{~m} / \mathrm{sec}(95 \% \mathrm{CI}$ of mean $=1.18-1.21$, range $1.08-1.21)$. Accordingly, the standardized speed greatly reduced the variability in walking speed. 
The mean kinematic errors differed by joint, segment, and plane (Figure 3). The greatest interlab kinematic errors occurred in both the sagittal and transverse planes with hip and knee flexion and hip rotation registering the largest errors. At the self-selected walking speed, the median inter-trial error was 1.0 degrees (ICR: 0.6-1.2 degrees, max: 1.7 degrees), the medial inter-session error was 1.2 degrees (ICR $1.1-1.7$ degrees, max: 2.5 degrees), and the median inter-lab error was only 2.3 degrees (ICR: 1.8-3.5, max: 4.7 degrees). Overall, kinematic errors were generally similar between the self-selected and Froude speeds and the same sagittal and transverse hip and knee rotations still registered the greatest inter-lab errors. At the Froude speed, the median inter-trial error was 0.9 degrees (ICR: 0.6-1.2, max: 1.5 degrees), the median inter-session error was 1.1 degrees (ICR: 1.0-1.6, max: 2.5 degrees), and the median inter-lab error was 2.3 degrees (ICR: 1.8-3.4, max: 4.7 degrees).

The kinetic error also varied considerably across joints, segments, and planes (Figure 4). At the self-selected speed, non-normalized errors in the hip rotation and knee flexion/extension moments were consistently greater than other values. The median inter-trial error at the self-selected speed was 0.088 $\mathrm{Nm} / \mathrm{kg}$ (ICR: $0.065-0.156, \max 0.158 \mathrm{Nm} / \mathrm{kg}$ ), the median inter-session error was $0.091 \mathrm{Nm} / \mathrm{kg}$ (ICR $0.067-0.167$, max: $0.168 \mathrm{Nm} / \mathrm{kg}$ ), and the median inter-lab error was $0.112 \mathrm{Nm} / \mathrm{kg}$ (ICR 0.091-0.184, max: $0.226 \mathrm{Nm} / \mathrm{kg}$ ). In order to facilitate interpretation, the kinetic errors were normalized to their peak kinetic values and expressed as a percentage of the normalized value. When this was done, hip and knee external/internal rotation moments were consistently greater than all other values. The median normalized inter-trial error was $20 \%$ (ICR 6 - 44\%, maximum 49\%), the median inter-session error was 22\% (ICR 6 - 45\%, maximum 53\%), and the median inter-lab error was 25\% (ICR 8 - 51\%, maximum $58 \%)$.

The kinetic errors were reduced by an average of $60 \%$ when subjects walked at their Froude speed, but errors still varied across joints, segments and planes. The greatest non-normalized inter-lab 
error was in the sagittal plane knee moment and the lowest was in the transverse plane knee moment. The median inter-trial error was $0.034 \mathrm{Nm} / \mathrm{kg}$ (ICR $0.013-0.038$, maximum 0.040 ), inter-session error was $0.033 \mathrm{Nm} / \mathrm{kg}$ (ICR $0.014-0.040$, maximum 0.040) and inter-lab error was $0.048 \mathrm{Nm} / \mathrm{kg}$ (ICR $0.025-0.078$, maximum 0.086). The normalized kinetic data for intra-trial (median $6 \%$, ICR $4-7 \%$, maximum 7\%), inter-session (median 6\%, ICR 5 - 7\%, maximum 8\%) and inter-lab (median 11\%, ICR $7-13 \%$, maximum 16\%) were much lower and within an acceptable range. Between labs, the greatest normalized error was still in the sagittal plane knee moment but the lowest was in the sagittal plane ankle moment.

\section{DISCUSSION}

The study results demonstrate that reliable gait data can be collected across multiple gait laboratories. In this study we compared the lower extremity kinematic and kinetic data collected on 10 healthy subjects who were studied in three gait laboratories. The three laboratories had different hardware configurations, marker configurations, and examiners that could have contributed to inconsistencies across the laboratories. The possible sources of variability were not controlled, simulating a real world scenario. However, the operational characteristics of each laboratory were carefully studied prior to data collection. This assured that all laboratories were operating at maximum capabilities. Further, the underlying anatomical model was consistent across all laboratories. This, most likely, contributed to the reliability of the gait data.

This is the first study to demonstrate acceptable repeatability across gait laboratories with multiple subjects. Previous studies have investigated inter-laboratory $[9,10]$ and inter-session [18] consistency of gait analysis measurements on a single subject and concluded that the variability in results can be attributed to differences in marker placement between examiners and trials. Other studies 
have confirmed that the repeatability of gait data depends on an accurate location of anatomical landmarks [19]. It was not possible to directly compare the results from this study to these previous studies because each study used different methodology for assessing reliability. The current study calculated the inter-lab standard deviation over the gait cycle to assess data reliability [2]. This method was selected because it could distribute the experimental error into known sources of variability in gait measurements. However, similarities do exist in these studies.

The goal was to collect kinematic data that differed by less than five degrees, which was the upper end of the minimal detectable change for almost all measures [20]. This was achieved across the planes of motion but the transverse plane was most variable. Benedetti et al. [7] also found that transverse plane rotations were the least consistent among laboratories $\left(\mathrm{r}^{2}=0.30\right)$, compared to sagittal and frontal plane rotations $\left(\mathrm{r}^{2}>0.70\right)$. In addition, the transverse plane rotations at the hip joint may be one of the parameters most affected by inter-lab variability as Gorton et al. [5] reported large deviations in hip rotation of 28.3 degrees across laboratories. Recently, Pinzone has suggested a method for harmonizing data collection protocols that reflect systematic differences in technique between two centers [21]. Using this approach it is possible to obtain inter-center differences of $<1.7^{\circ}$ for all kinematic variables except hip rotation $\left(2.9^{\circ}\right)$ and foot progression $\left(2.1^{\circ}\right)$. In the present study, we also found transverse plane hip rotation consistently exhibited the greatest kinematic variability among laboratories and also across sessions.

There were differences in gait data reliability for the self-selected walking speed vs. the standardized walking speed, i.e. Froude speed. The data were more consistent for the standardized walking speed, and the error of the kinetic parameters were particularly reduced. Walking speed is an important consideration in clinical gait studies as individuals with pathology typically walk slower than healthy, able-bodied individuals [22, 23], and walking speed is often considered a reflection of overall 
muscle function. Subjects are often studied at their self-selected walking speed because it is thought that subjects adopt walking speed which is most energy efficient [24]. Further, the self-selected speed normalizes for age and physical factors [25]. However, significant associations between speed and gaitrelated variables have been found in both healthy and subjects with pathology [26-29], which creates an inherent problem in the interpretation of gait analysis results. Therefore, if the goal is to obtain reliable gait data across trials, sessions, and laboratories, it is important to standardize walking speed.

Establishing a standardized walking speed with Froude numbers is useful because it accounts for inter-subject differences in geometry by adjusting walking speed by leg length, with individuals with shorter legs walking slower than individuals with longer legs. This method of standardization abides by the principle of dynamic similarity which states that the most economical walking speeds are independent of body geometry when speed is normalized to a dimensionless Froude number [17, 30$].$ The speed dependence of the gait parameters is reflected by the fact that the kinetic errors were substantially lower when the subjects walked at the standardized Froude speed than when they walked their self-selected walking speed. This was a direct result of the fact that the variability of the walking speed was greatly reduced when the subjects walked at a standardized walking speed as compared to a self-selected walking speed. We chose not to control the self-selected walking speed in this study to better capture how data collections would typically be performed in these three labs (and in most other labs).There was more variability in the self-selected walking speed, which is due to the height variation of the subjects. The variability was reduced with the Froude speed because we only accepted trials where the speed fell within $5 \%$ of the intended value. For the self-selected condition we accepted any walking speed and made no effort to accept trials that fell close to the mean self-selected walking speed. It can be reasonably expected that if we controlled self-selected speed and only accepted values that fell 
within a 5\% range, variability would decrease and the results would be much closer to the Froude speed results.

The limitation of this study is that it has been performed in a relatively homogenous sample of young healthy adults who would not require a gait analysis for clinical decision making purposes. Gait analysis studies are often performed in children or in patients with musculoskeletal pathology. In order to make the results more generalizable, this study will need to be repeated in young, healthy children and subjects with musculoskeletal pathology. Further studies are needed to evaluate reliability of gait data in patients with antalgic gait such as osteoarthritis and neuromuscular deficits such as cerebral palsy.

Data reliability is of utmost importance when attempting to share data across sites to evaluate patient progress or to pool research data for comparative effectiveness studies. Measurement consistency across sites participating in multi-center research studies directly influences required sample size, level of detectable difference, statistical power, and ultimately, the ability to detect real change. This study demonstrates that it is possible to obtain high quality, reliable data across multiple gait laboratories, even when using different marker placements, marker sets, examiners, collection environments, and instrumentation from different manufacturers. A key similarity among sites was the use of identical anatomical segment definitions for the respective gait models. The results of this study strongly support the importance of standardizing gait speed to ensure the most reliable data is being collected across sites, sessions, and trials.

\section{DISCLAIMER}


The views expressed in the article are those of the authors and do not reflect the official policy of Department of the Navy, Department of the Army, Department of Defense, or the US Government.

\section{ACKNOWLEDGEMENTS}

Funding was provided by DOD 731743-1, DOD Defense Health Programs/Center for Rehabilitative

Sciences Research, Grant \# HU0001-11-1-0004. There are no commercial relationships which may lead to a conflict of interest with any of the authors. 


\section{REFERENCES}

[1] Monaghan K, Delahunt E, Caulfield B. Increasing the number of gait trial recordings maximises intra-rater reliability of the CODA motion analysis system. Gait \& Posture. 2007;25:303-15.

[2] Schwartz MH, Trost JP, Wervey RA. Measurement and Management of Errors in Quantitative Gait Data. Gait \& Posture. 2004;20:196.

[3] Al-Obaidi S, Wall JC, Al-Yaqoub A, Al-Ghanim M. Basic gait parameters: a comparison of reference data for normal subjects 20 to 29 years of age from Kuwait and Scandinavia. Journal of Rehabilitation Research \& Development. 2003;40:361-6.

[4] Stansfield BW, Hillman SJ, Hazlewood ME, Lawson AA, Mann AM, Loudon IR, et al. Sagittal joint kinematics, moments, and powers are predominantly characterized by speed of progression, not age, in normal children. J Pediatr Orthop. 2001;21:403-11.

[5] Sutherland DH. The evolution of clinical gait analysis part III--kinetics and energy assessment. Gait \& Posture. 2005;21:447-61.

[6] Diss CE. The reliability of kinetic and kinematic variables used to analyse normal running gait. Gait \& Posture. 2001;14:98-103.

[7] Maynard V, Bakheit AM, Oldham J, Freeman J. Intra-rater and inter-rater reliability of gait measurements with CODA mpx30 motion analysis system. Gait \& Posture. 2003;17:59-67.

[8] Noonan KJ, Halliday S, Browne R, O'Brien S, Kayes K, Feinberg J. Interobserver variability of gait analysis in patients with cerebral palsy. J Pediatr Orthop. 2003;23:279-87; discussion 88-91.

[9] Gorton GE, Hebert DA, Gannotti ME. Assessment of the kinematic variability among 12 motion analysis laboratories. Gait \& Posture. 2009;29:398-402.

[10] Benedetti MG, Merlo A, Leardini A. Inter-laboratory consistency of gait analysis measurements. Gait \& Posture. 2013;38:934-9. 
[11] Piazza SJ, Chou L-S, Denniston NL, McMulkin ML, Quigley EJ, Richards JG, et al. A proposed standard for assessing the marker location accuracy of video-based motion analysis system. 12th Annual GCMAS. Springfield, MA2007.

[12] Holden JP, Selbie WS, Stanhope SJ. A proposed test to support the clinical movement analysis laboratory accreditation process. Gait \& Posture. 2003;17:205-13.

[13] Kadaba M, Ramakrishnan H, Wootten M. Measurement of lower extremity kinematics during level walking. Journal of Orthopedic Research. 1990;8:383-92.

[14] Manal K, McClay Davis I, Galinat B, Stanhope S. The accuracy of estimating proximal tibial translation during natural cadence walking: bone vs. skin mounted targets. Clinical Biomechanics. 2003; 18:126-31.

[15] Bell AL, Pedersen DR, Brand RA. A comparison of the accuracy of several hip center location prediction methods. J Biomech. 1990;23:617-21.

[16] Bell AL, Pederson DR, Brand RA. Prediction of hip joint center location from external landmarks. Human Movement Science. 1989;8:3-16.

[17] Alexander RM. Optimization and gaits in the locomotion of vertebrates. Physiol Rev. 1989;69:1199-227.

[18] Growney E, Meglan D, Johnson M, Cahalan T, An K-N. Repeated measures of adult normal walking using a video tracking system. Gait and Posture. 1997;6:147-62.

[19] Besier TF, Sturnieks DL, Alderson JA, Lloyd DG. Repeatability of gait data using a functional hip joint centre and a mean helical knee axis. J Biomech. 2003;36:1159-68.

[20] Wilken JM, Rodriguez KM, Brawner M, Darter BJ. Reliability and Minimal Detectible Change values for gait kinematics and kinetics in healthy adults. Gait Posture. 2012;35:301-7. 
[21] Pinzone O, Schwartz MH, Thomason P, Baker R. The comparison of normative reference data from different gait analysis services. Gait \& Posture. 2014;40:286-90.

[22] Kaufman KR, Hughes C, Morrey BF, Morrey M, An KN. Gait characteristics of patients with knee osteoarthritis. J Biomech. 2001;34:907-15.

[23] Olney SJ, Griffin MP, McBride ID. Temporal, kinematic, and kinetic variables related to gait speed in subjects with hemiplegia: a regression approach. Phys Ther. 1994;74:872-85.

[24] Ralston HJ. Energy-speed relation and optimal speed during level walking. Int $\mathrm{Z}$ angew Physiol einschl Arbeitsphysiol. 1958;Bd. 17:S.277-83.

[25] DeVita P, Hortobagyi T. Age causes a redistribution of joint torques and powers during gait. J Appl Physiol. 2000;88:1804-11.

[26] Chiu M-C, Wang M-J. The effect of gait speed and gender on perceived exertion, muscle activity, joint motion of lower extremity, ground reaction force and heart rate during normal walking. Gait and Posture. 2006;25:385-92.

[27] Chung MJ, Wang MJ. The change of gait parameters during walking at different percentage of preferred walking speed for healthy adults aged 20-60 years. Gait \& Posture. 2010;31:131-5. [28] Mockel G, Perka C, Labs K, Duda G. The influence of walking speed on kinetic and kinematic parameters in patients with osteoarthritis of the hip using a force-instrumented treadmill and standardised gait speeds. Archives of Orthopaedic \& Trauma Surgery. 2003;123:278-82.

[29] Zeni JAJ, Higginson JS. Differences in gait parameters between healthy subjects and persons with moderate and severe knee osteoarthritis: A result of altered walking speed? Clinical Biomechanics. $2009 ; 24: 372-8$.

[30] Vaughan CL, O'Malley MJ. Froude and the contribution of naval architecture to our understanding of bipedal locomotion. Gait \& Posture. 2005;21:350-62. 
Table 1. Gait Lab systems used in the study

\begin{tabular}{|c|c|c|c|c|}
\hline & & MTF1 -- NMCSD & MTF2 -- CFI & MTF3 -- WR NMMC \\
\hline \multirow{4}{*}{$\begin{array}{l}\text { Motion } \\
\text { Capture } \\
\text { System }\end{array}$} & Manufacturer & $\begin{array}{l}\text { Motion Analysis Corp } \\
\text { (Santa Rosa, CA) }\end{array}$ & $\begin{array}{l}\text { Motion Analysis Corp } \\
\text { (Santa Rosa, CA) }\end{array}$ & $\begin{array}{c}\text { Vicon } \\
\text { (Oxford, UK) }\end{array}$ \\
\hline & Camera & Eagle & Eagle and Raptor-E & $\mathrm{MX}$ \\
\hline & Number of Cameras & 12 & 26 & 26 \\
\hline & Data Collection Rate (Hz) & 120 & 120 & 120 \\
\hline \multirow{4}{*}{$\begin{array}{l}\text { Force } \\
\text { Plates }\end{array}$} & Manufacturer & $\begin{array}{c}\text { AMTI } \\
\text { (Watertown, MA) }\end{array}$ & $\begin{array}{c}\text { AMTI } \\
\text { (Watertown, MA) }\end{array}$ & $\begin{array}{c}\text { AMTI } \\
\text { (Watertown, MA) }\end{array}$ \\
\hline & Model & OR6-7-1000 & BP600600-2000 & $\begin{array}{c}\text { BP6001200 (2) } \\
\text { OR6-7 (4) }\end{array}$ \\
\hline & Number of Plates & 4 & 5 & 6 \\
\hline & Data Collection Rate (Hz) & 1800 & 600 & 1200 \\
\hline
\end{tabular}


Figure 1. Lower extremity tracking marker configurations used in the study. A modified Helen Hayes model was used by the MTF 1 (NMCSD) (Figure 1a). A modified Cleveland Clinic marker set was used by MTF 2 (CFI) and MTF 3 (WRNMMC) (Figure 1b). However, CFI is the only lab that used the Iliac Crest markers. The black circles are physical markers. The open circles are calibration points. MTF 1 and MTF 3 used physical makers at these locations while MTF 2 used a pointer stick to digitize the location. For all models, the location of the hip joint center (HJC) was calculated from the left and right anterior superior iliac spine (ASIS) markers and the sacrum marker (Figure 1a) or the midpoint of the of the posterior superior iliac spines (PSIS) markers (Figure 1b) using the Bell hip model [15, 16]. The knee joint center was defined as the midpoint between the medial and lateral femoral epicondyles. The ankle joint center was defined as the midpoint between the medial and lateral ankle malleoli. All makers were used to calculate gait kinematics and kinetics.

Figure 2. Reliability data for the (a) kinematics and (b) kinetics of a single subject walking at their selfselected walking speed. Average data are shown at each point across the gait cycle.

Figure 3. Inter-trial, inter-session, and inter-lab kinematic error for all subjects walking at their (a) selfselected and (b) Froude walking speeds.

Figure 4. Inter-trial, inter-session, and inter-lab kinetic error for all subjects walking at their (a) selfselected and (b) Froude walking speeds. The error is reported in both absolute terms $(\mathrm{Nm} / \mathrm{kg})$ and normalized $(\%)$ to the maximum moment during gait. 


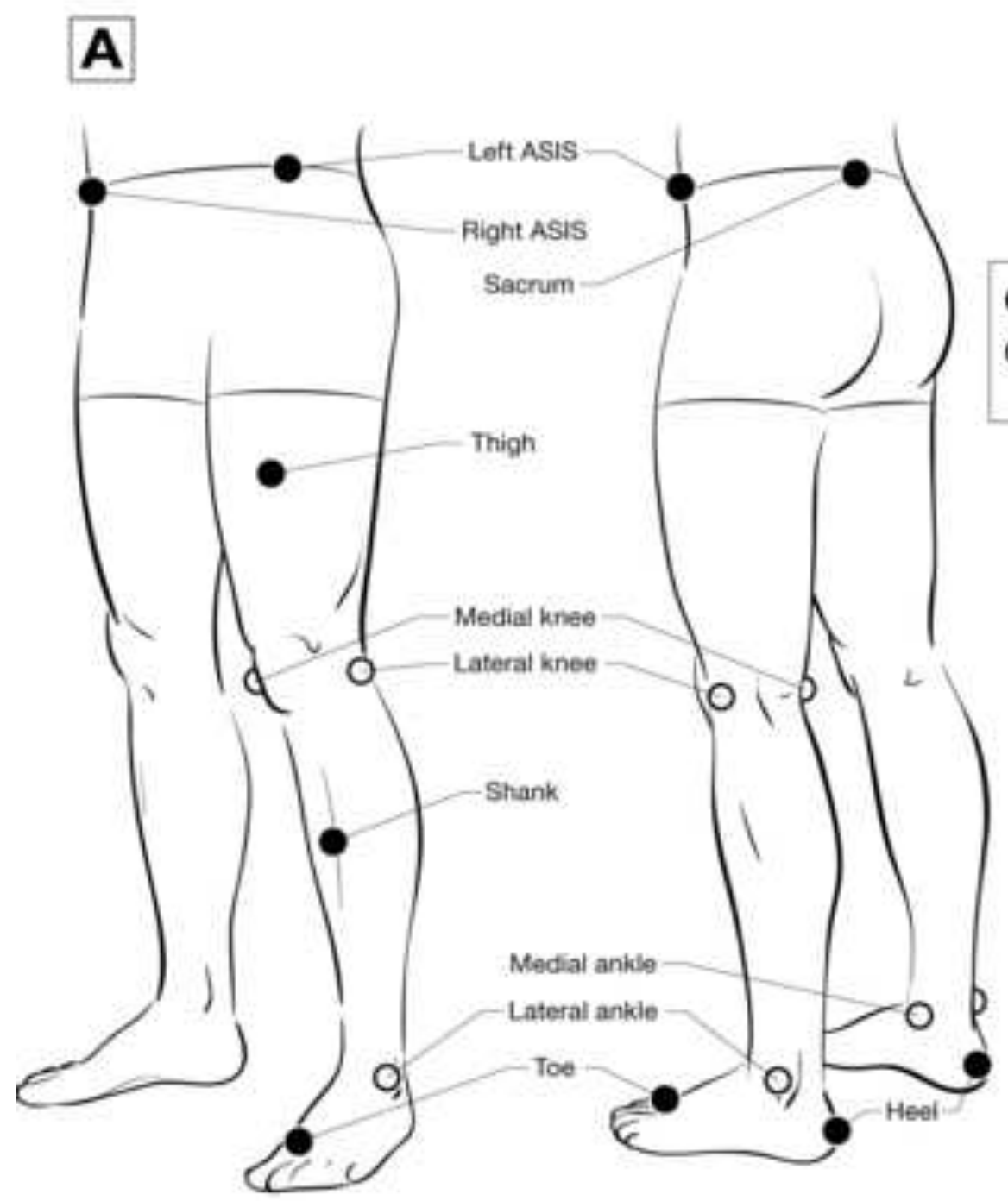

\section{B}

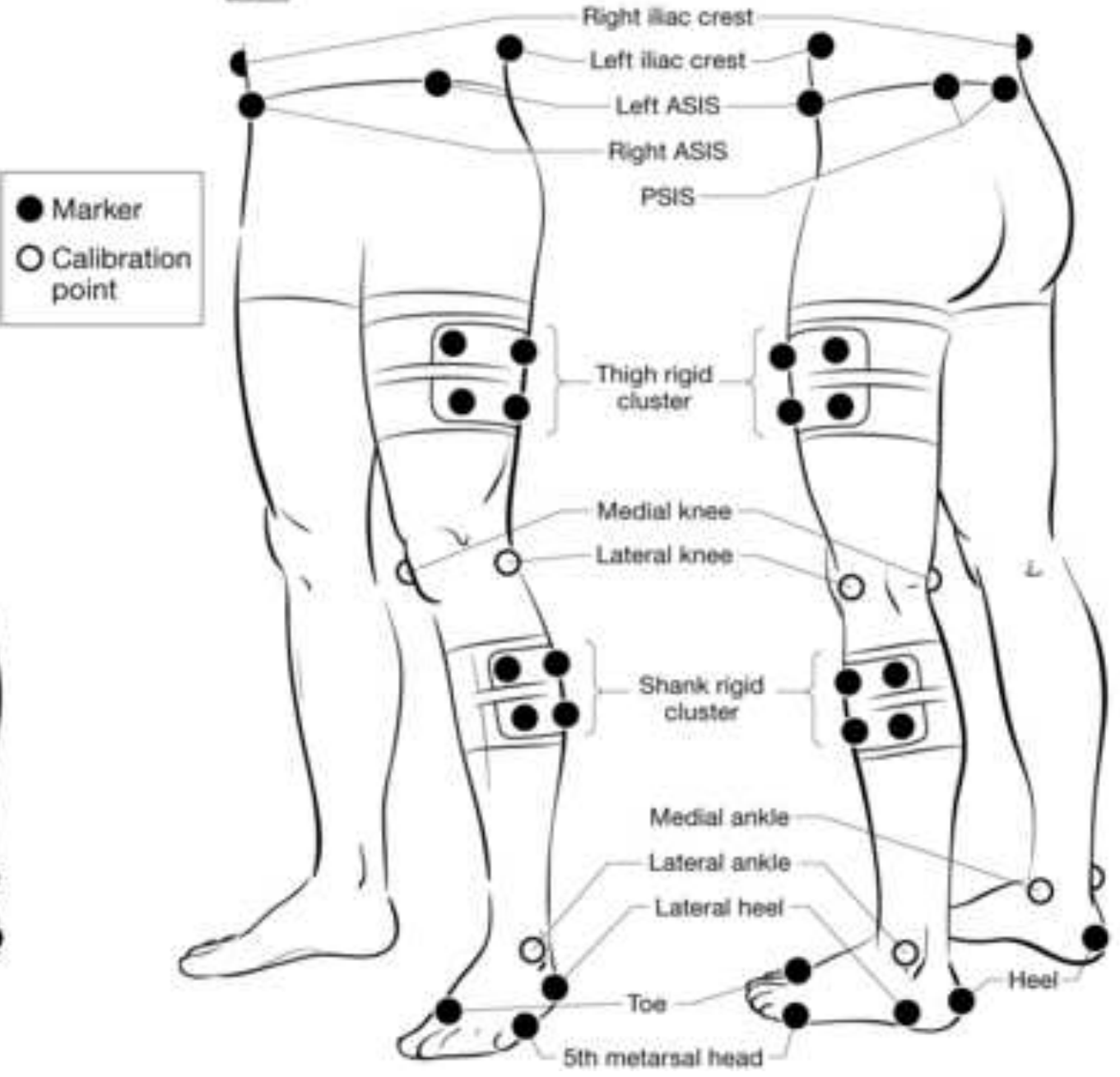




\section{KINEMATICS}
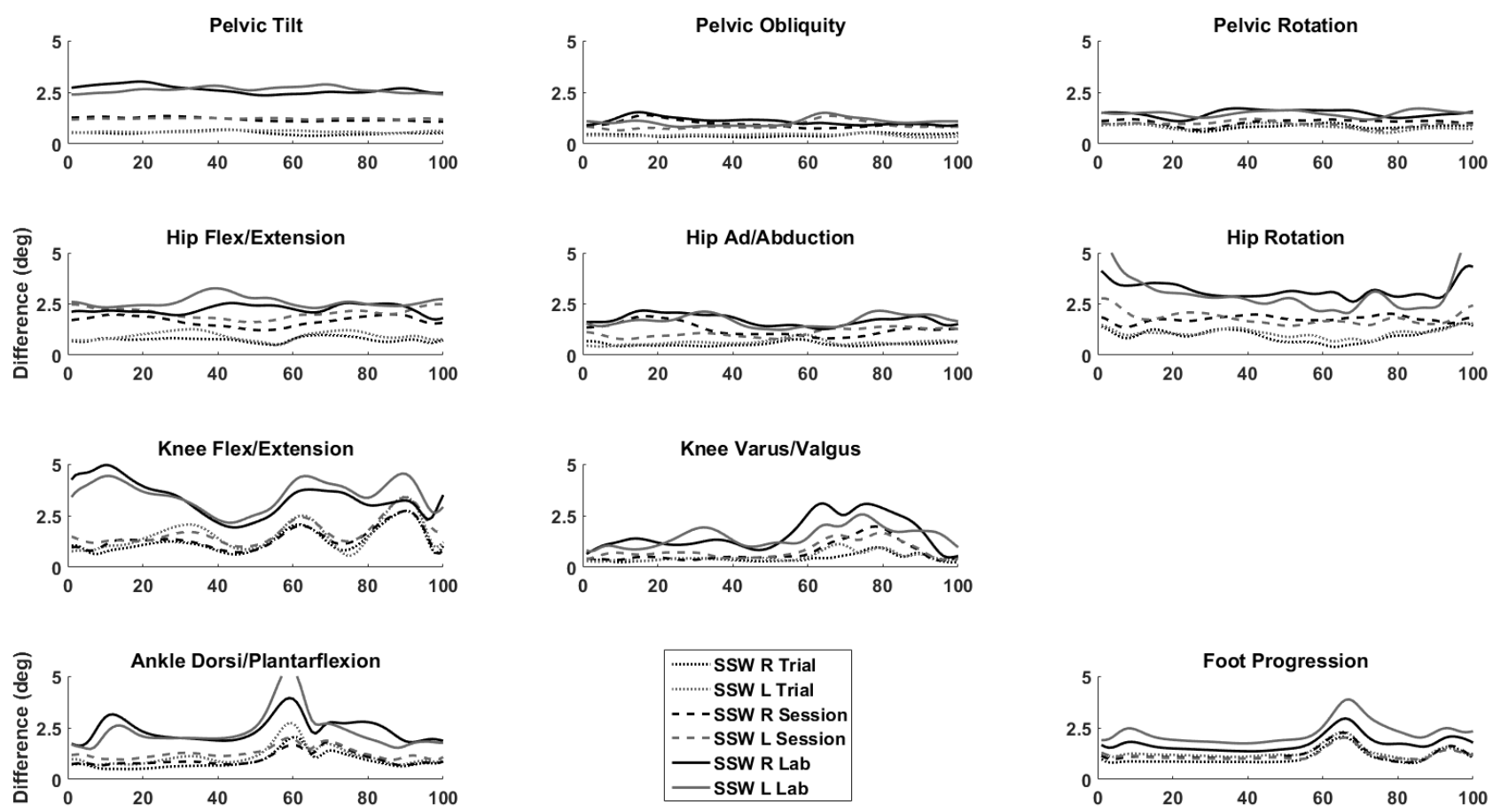

\section{KINETICS}
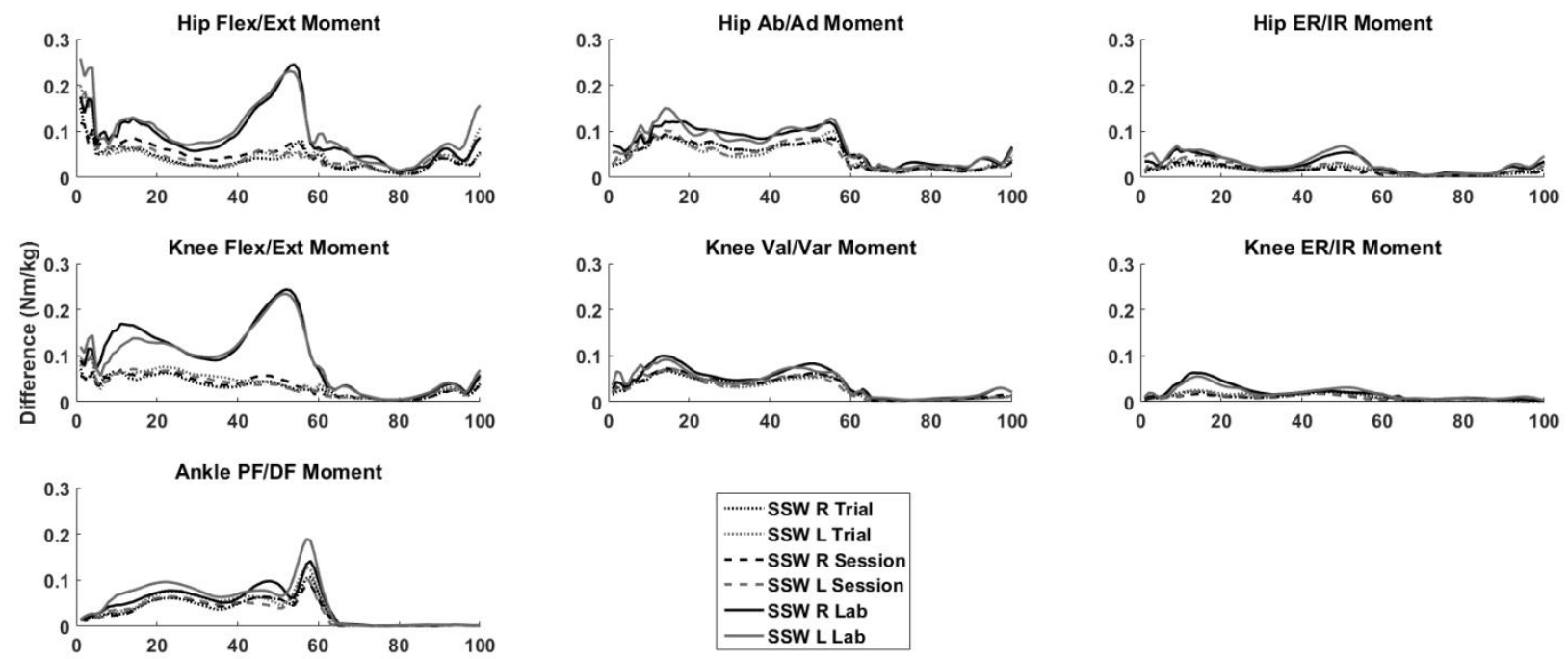

Figure 2. Reliability data for the (a) kinematics and (b) kinetics of a single subject walking at their self-selected walking speed. Average data are shown at each point across the gait cycle. 
Self-selected walking speed

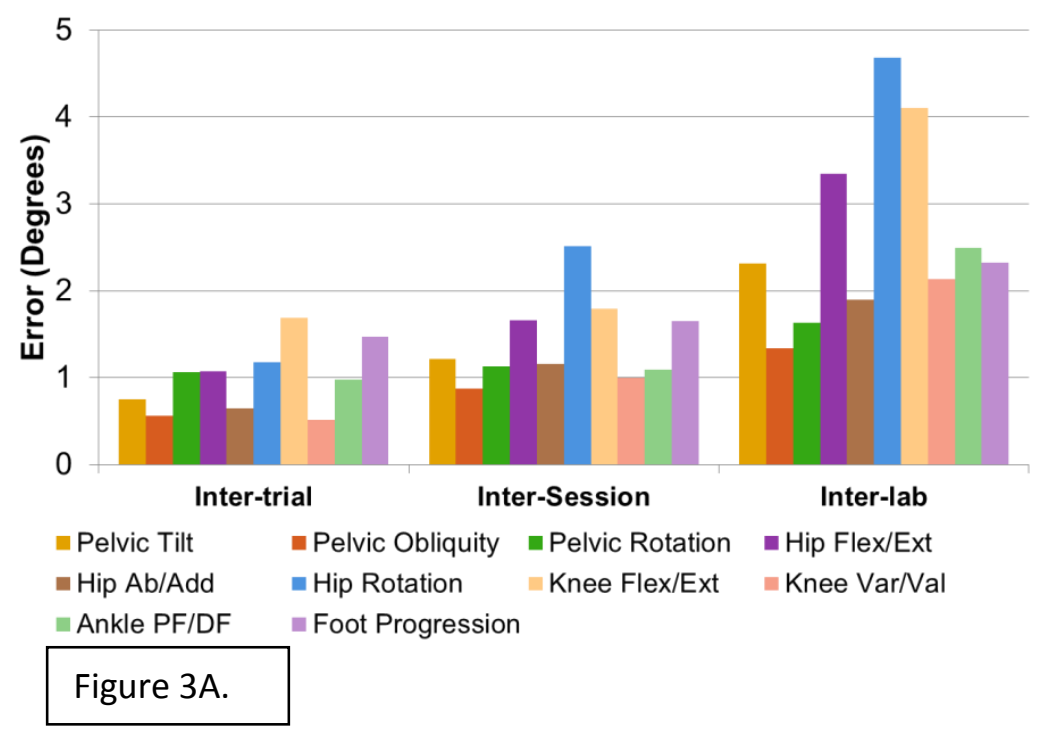

\section{Froude Speed}

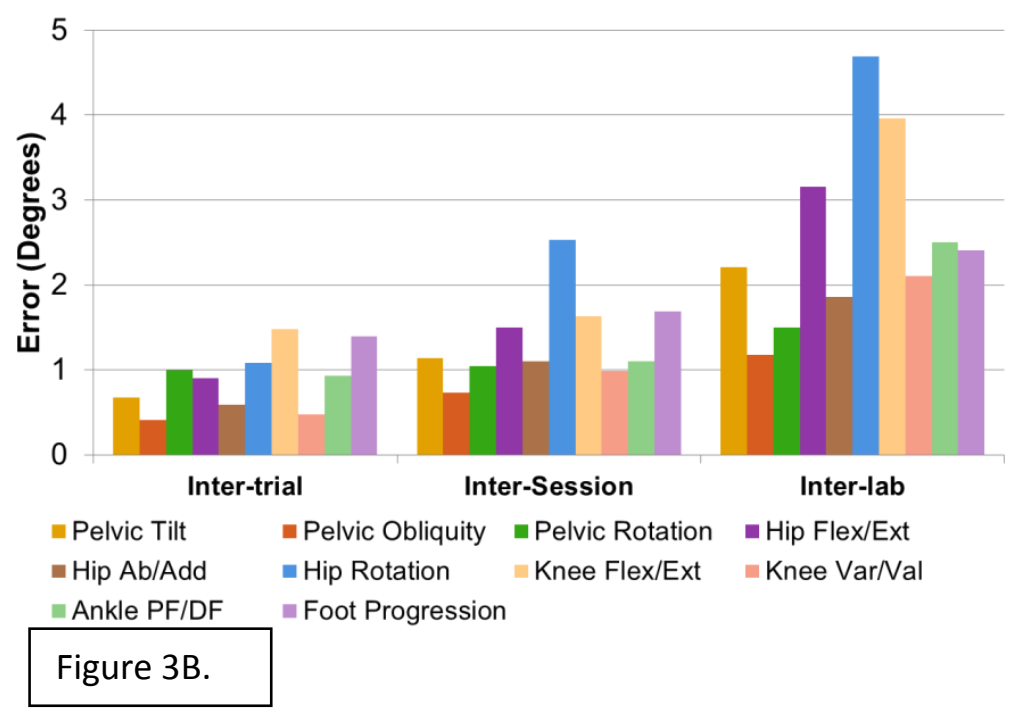



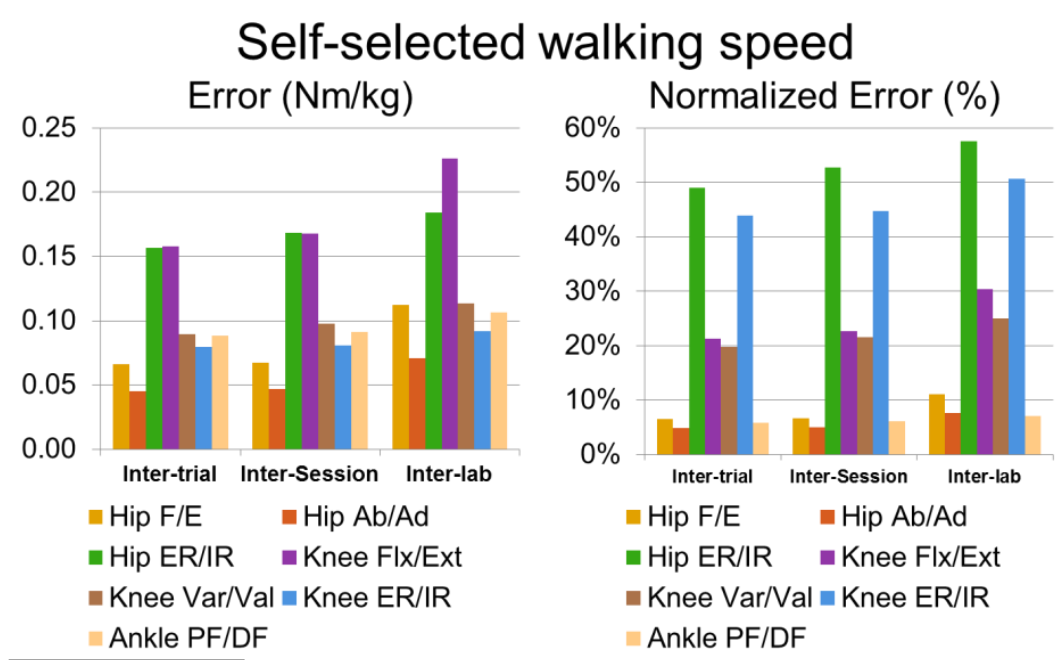

Figure 4A.

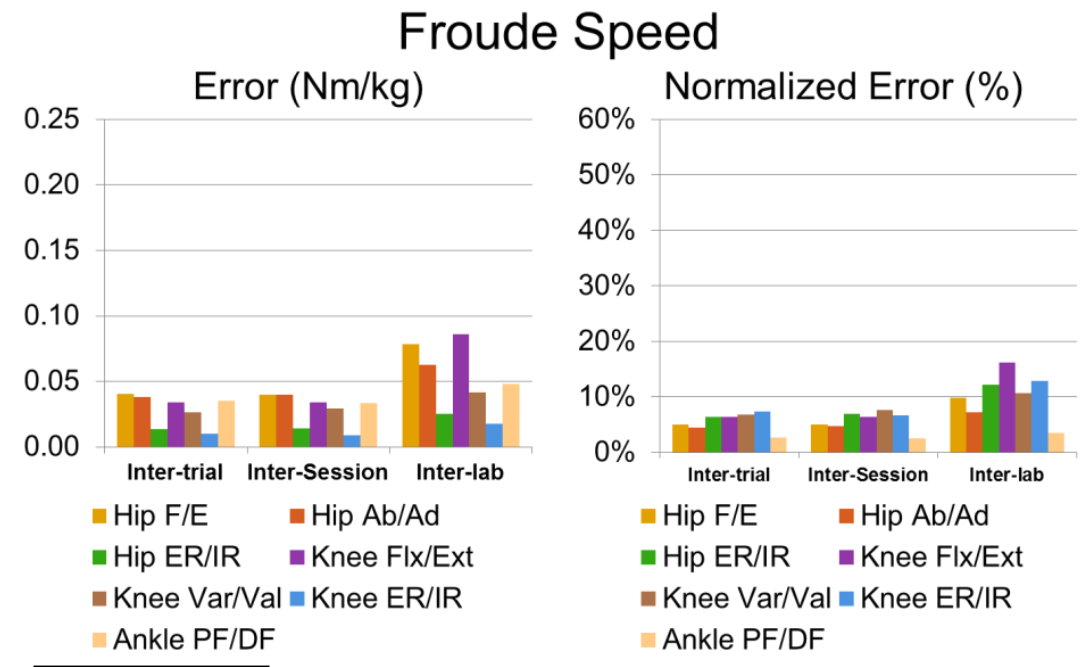

Figure 4B. 